\title{
Inhibition of DNA replication in Staphylococcus aureus by tegaserod
}

\author{
Christopher J Lacriola, Shaun P Falk and Bernard Weisblum \\ The Journal of Antibiotics (2017) 70, 918-920; doi:10.1038/ja.2017.65; published online 31 May 2017
}

DNA replication is a vital function for cellular growth and proliferation. Enzymes that support this process would serve as useful targets for antibiotic discovery. Here we describe the antibacterial activity of tegaserod (TG) and propose a mechanism of action based on inhibition of Staphylococcus aureus DnaG primase, a DNA-dependent RNA polymerase required for the initiation of replication. TG, whose structure is shown in Figure 1a, was originally developed for clinical use as a 5HT-4 (serotonin) receptor agonist to treat gastrointestinal disorders. ${ }^{1}$ In the studies described below, TG was, additionally, identified as a hit in a DnaG screen (Profoldin, Hudson, MA, USA) of a library of 450 previously approved drugs (NIH CC1 collection). The screen is based on the measurement of fluorescence enhancement of an intercalating dye into RNA-DNA heteroduplexes synthesized by DnaG. Library compounds were tested at a final concentration of $30 \mu \mathrm{m}$ in a $20 \mu \mathrm{l}$ reaction volume. Inhibition was defined as a fluorescence reduction $>3$ s.d.'s compared to untreated control. The identified inhibitors of DnaG, together with their respective percent inhibition in the screen, included: doxorubicin (71\%), epirubicin (98\%), TG (71\%), epigallocathechin gallate $(58 \%)$, pterostilbene (52\%), actinomycin D (52\%) and nonyloxyltryptamine (50\%). With the exception of TG, the hits that were obtained in the screen had been studied elsewhere as inhibitors of nucleic acid metabolism. ${ }^{2-6}$ We therefore focused our attention on TG for further characterization of its antimicrobial activity.

To confirm these results, a polymerization assay based on the work of Koepsell et al..$^{7}$ in which the 37-mer single stranded DNA template $5^{\prime}$-CAG(AC) ${ }_{12}$ ACTACACACA-3' (transcription initiation start site at $\mathrm{T}$, coordinate 30 , underlined) was used. The known inhibitor of DnaG primase activity, doxorubicin, ${ }^{4}$ was included as a positive control for these studies (Figure 1b, Lane 1). The complete in vitro reaction was supplemented with an incremental series of TG concentrations. Polymerization products labeled by inclusion of $\left[\alpha-{ }_{-}{ }^{32} \mathrm{P}\right]$ UTP in the reaction mix were fractionated by PAGE and analyzed by autoradiography. ${ }^{8}$ The results, Figure $1 \mathrm{~b}$, lanes 2 and 3, show the expected 30-mer RNA primer that is formed in the complete uninhibited reaction. Results shown in lanes 4, 5 and 6, indicate that TG inhibits RNA primer formation in a TG-dependent manner with nearly complete inhibition of 30 -mer transcript formation at $20 \mu \mathrm{M}$, near the MIC. Lanes 6 and 7 show the reappearance of transcript formation and a shift of the transcription product length to $60 \mathrm{nt}$. The 60-mer product may correspond to RNA formed by extension of the 37-mer template beginning at its $3^{\prime} \mathrm{OH}$, and it is similar to the overlong product seen in earlier reports. ${ }^{9}$ We postulate that the $60-\mathrm{nt}$ product is an RNA-DNA heteroduplex hairpin loop in which $5^{\prime}$-TACACACA-3' forms the loop portion by association of the $5^{\prime} \mathrm{T}$ with the $3^{\prime} \mathrm{A}$. Lane 8 shows the products formed by DnaG in the presence of $100 \mu \mathrm{M}$ TG-complete abolition of 30-mer synthesis and marked reduction in 60-mer synthesis.

To compare the specificity of DnaG with that of other polymerases, four additional enzymes were tested, namely, Escherichia coli DNA-dependent RNA polymerase and E. coli PolA (NEB, Ipswich, MA, USA); T7 DNA-dependent RNA polymerase (Fermentas, Waltham, MA, USA); and MuLV reverse transcriptase (Thermo Fisher, Fitchburg, WI, USA). The DNA templates used for the four enzymes were, calf thymus DNA, DNAase-treated calf thymus DNA, PCR-amplified T7 polymerase promoter sequence, and Bacillus. subtilis total RNA, respectively. Polymerase reactions were performed based on protocols provided by the vendor.

Results, shown in Supplementary Figure 1, indicate that all four polymerases along with DnaG were inhibited by aurintricarboxylic acid, a non-specific inhibitor of nucleic acid-binding proteins. ${ }^{10}$ Tested at a maximum concentration of $200 \mu \mathrm{M}$, doxorubicin selectively inhibited the two RNA polymerases, but not the DNA polymerases PolA or MuLV reverse transcriptase. Importantly, none of the polymerases were inhibited by TG. TG, therefore, appears to have a unique mode of action in its ability to inhibit the polymerase activity of DnaG.

To test the susceptibility of S. aureus 1206 cells $^{11}$ in vivo, we determined the MIC $(25 \mu \mathrm{M})$ and the MBC $(100 \mu \mathrm{M})$ for TG. We measured the effect of TG on DNA, RNA and protein synthesis at the MIC using intact cells. Mid-exponential cultures of S. aureus 1206 in LB medium were supplemented with ${ }^{3} \mathrm{H}$-thymidine, ${ }^{3} \mathrm{H}$-uracil or ${ }^{3} \mathrm{H}$-tyrosine followed by a challenge with TG or control antibiotic for $1 \mathrm{~h}$. Results, shown in Figure 2a, indicate that TG at a concentration 
a

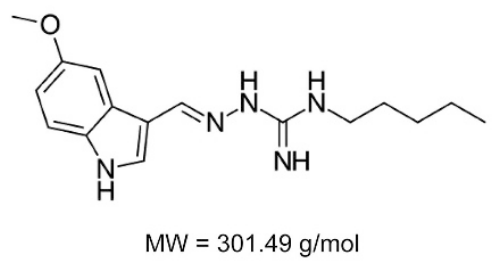

b

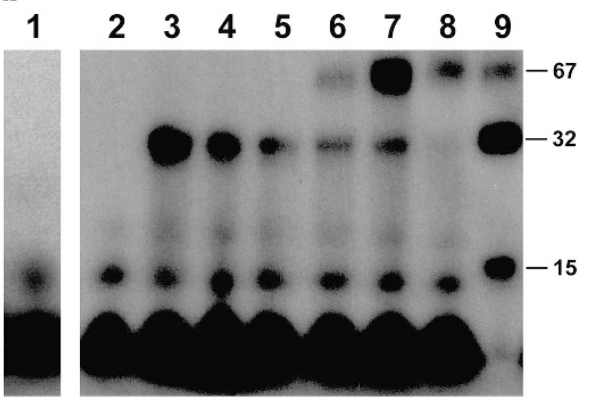

Figure 1 RNA primers synthesized in the presence of TG in vitro. (a) Chemical structure of TG. (b) S. aureus DnaG primase incubated with increasing concentrations of TG. RNA primer product was visualized by $\left[\alpha^{-32}\right.$ P]UTP incorporation and fractionation of the resultant products by urea-PAGE separation.

(1) Complete plus $50 \mu \mathrm{m}$ Doxorubicin; (2) Complete minus $37 \mathrm{nt} \mathrm{ssDNA}$ template; (3) Complete; (4) Complete plus $5 \mu \mathrm{m}$ TG; (5) Complete plus $10 \mu \mathrm{m}$ TG;

(6) Complete plus $20 \mu \mathrm{m}$ TG; (7) Complete plus $50 \mu \mathrm{m} \mathrm{TG;} \mathrm{(8)} \mathrm{Complete} \mathrm{plus} 100 \mu \mathrm{m}$ TG; (9) Ladder (15, 32, 67 nucleotide standards).

\section{a}
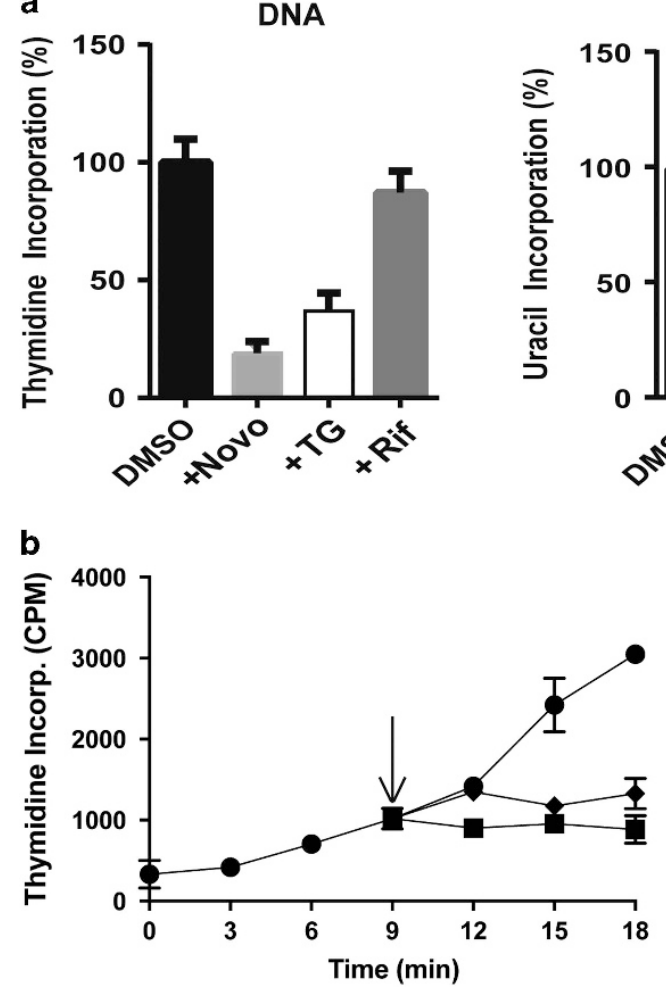

RNA

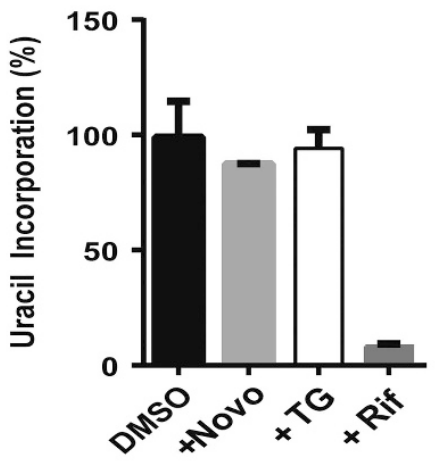

Protein

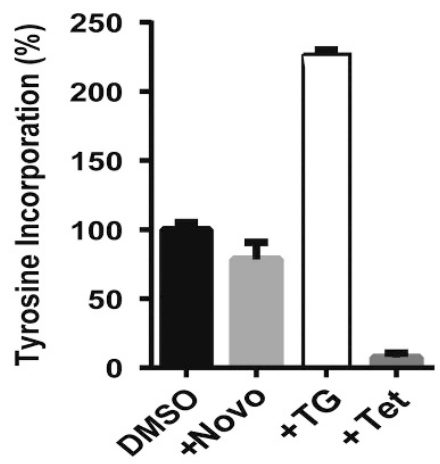

C

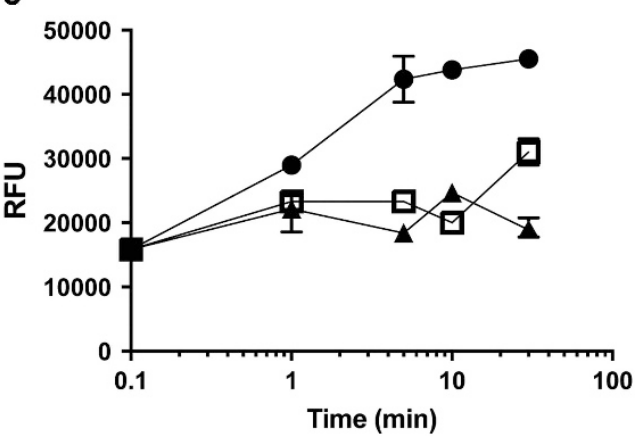

Figure 2 TG inhibits DNA replication and membrane integrity in vivo. (a) The susceptibility of methicillin-resistant clinical isolate S. aureus 1206 cell growth to inhibitors of DNA, RNA, and protein synthesis measured as thymidine incorporation (left panel) uracil incorporation (center panel), tyrosine incorporation (right panel) with $5 \%$ DMSO as control; Novo, novobiocin $50 \mu \mathrm{m}$; Rif, rifampicin $25 \mu \mathrm{m}$; TG, $25 \mu \mathrm{m}$; Tet, tetracycline $25 \mu \mathrm{m}$. (b) Thymidine incorporation was

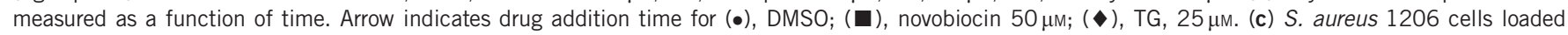
with DiSC3(5) were exposed to TG $(\mathbf{\Lambda}), 25 \mu \mathrm{m} ;(\square), 50 \mu \mathrm{m} ;(\bullet), 100 \mu \mathrm{m}$ over $20 \mathrm{~min}$ and depolarization of the membrane was recorded as an increase fluorescence.

of $25 \mu \mathrm{m}$ inhibited DNA synthesis by $70 \%$, while under the same growth conditions, RNA synthesis was inhibited by only $10 \%$, and protein synthesis was actually stimulated by $130 \%$. Lastly, measurement of thymidine incorporation as a function of time showed that the inhibitory activity of TG was expressed within $3 \mathrm{~min}$ after addition (Figure 2b).

As to the chemical structure of TG, hydrophobicity of the pentyl side chain, (Figure 1a), raised concerns of its possible aggregation in the cell membrane, leading to membrane lysis and consequent depolarization.
Maintenance of the membrane potential in S. aureus 1206 incubated with TG was tested using the fluorescent potentiometric dye DiSC3 (5). ${ }^{12}$ Results, shown in Figure $2 c$, indicate that membrane depolarization by TG occurs at $100 \mu \mathrm{m}$ but not at $25 \mu \mathrm{m}$. Since DNA replication is inhibited at concentrations below those perturbing the membrane, we infer that the primary effect of TG is on DNA synthesis.

Lastly, tests of TG susceptibility of various bacterial strains showed growth inhibition of $B$. subtilis and Enterococcus faecium with $\mathrm{MIC}=12 \mu \mathrm{M}$, while an $\mathrm{MIC}=50 \mu \mathrm{M}$ was determined for $E$. coli and 
Salmonella enterica. The range of susceptibilities of several bacterial strains is presented in Supplementary Table 1.

In summary, TG, at a concentration of $25 \mu \mathrm{M}$, inhibits DNA replication in vivo, and DnaG primase in vitro. It is active against both Gram-positive and Gram-negative bacteria. Results from in vitro experiments support a model according to which TG inhibits an early step of DNA replication, namely, RNA primer synthesis by DnaG, but is without effect on four other nucleotide polymerases in vitro. It will be interesting to learn how the antimicrobial activity of TG affects the intestinal microbiome.

\section{CONFLICT OF INTEREST}

The authors declare no conflict of interest.

\section{ACKNOWLEDGEMENTS}

We thank James Berger and Steven Hinrichs for bacterial strains used to produce recombinant proteins; Jim Keck and Jade Wang for helpful discussions, Karen Wassarman, for assistance with primase product fractionation; Megan Duster for assistance with bacterial susceptibility studies For assistance with screening, we thank Gene Ananiev and the University of Wisconsin Small Molecule Screening Facility supported by University of Wisconsin Carbone Cancer Center support grant P30 CA014520.
1 Beglinger, C. Tegaserod: a novel, selective 5-HT4 receptor partial agonist for irritable bowel syndrome. Int. J. Clin. Pract. 56, 47-51 (2002).

2 Agarwal, A. et al. Small molecule inhibitors of $E$. coli primase, a novel bacterial target. Bioorg. Med. Chem. Lett. 17, 2807-2810 (2007).

3 Biswas, T., Green, K. D., Garneau-Tsodikova, S. \& Tsodikov, O. V. Discovery of inhibitors of Bacillus anthracis primase DnaG. Biochemistry 52, 6905-6910 (2013).

4 Gajadeera, C. et al. Antimycobacterial activity of DNA intercalator inhibitors of Mycobacterium tuberculosis primase DnaG. J. Antibiot. 68, 153-157 (2015).

5 Sanyal, G. \& Doig, P. Bacterial DNA replication enzymes as targets for antibacterial drug discovery. Expert Opin. Drug Discov. 7, 327-339 (2012).

6 Zhang, Y. et al. Homogenous assays for Escherichia coli DnaB-stimulated DnaG primase and DnaB helicase and their use in screening for chemical inhibitors. Anal. Biochem. 304, 174-179 (2002).

7 Koepsell, S. A., Larson, M. A., Frey, C. A., Hinrichs, S. H. \& Griep, M. A. Staphylococcus aureus primase has higher initiation specificity, interacts with single-stranded DNA stronger, but is less stimulated by its helicase than Escherichia coli primase. Mol. Microbiol. 68, 1570-1582 (2008).

8 Swart, J. R. \& Griep, M. A. Primer synthesis kinetics by Escherichia coli primase on single-stranded DNA templates. Biochemistry 34, 16097-16106 (1995).

9 Johnson, S. K., Bhattacharyya, S. \& Griep, M. A. DnaB helicase stimulates primer synthesis activity on short oligonucleotide templates. Biochemistry 9, 736-744 (2000).

10 Shadrick, W. R., Mukherjee, S., Hanson, A. M., Sweeney, N. L. \& Frick, D. N. Aurintricarboxylic acid modulates the affinity of hepatitis C virus NS3 helicase for both nucleic acid and ATP. Biochemistry 52, 6151-6159 (2013).

11 Weisblum, B. \& Demohn, V. Erythromycin-inducible resistance in Staphylococcus aureus: survey of antibiotic classes involved. J. Bacteriol. 98, 447-452 (1969).

12 Hobbs, J. K., Miller, K., O'Neill, A. J. \& Chopra, I. Consequences of daptomycinmediated membrane damage in Staphylococcus aureus. J. Antimicrob. Chemother. 62, 1003-1008 (2008)

Supplementary Information accompanies the paper on The Journal of Antibiotics website (http://www.nature.com/ja) 